\title{
Design of 5.83GHz Microstrip Antenna Used in the OBU
}

\author{
Yang Cheng-xiang \\ College of Information Engineering, \\ North China University of Technology, \\ Beijing, China \\ E-mail:ycx621@sina.com
}

\author{
Guan Xiao-han \\ College of Information Engineering, \\ North China University of Technology, \\ Beijing, China \\ E-mail: gxh@ncut.edu.cn
}

\begin{abstract}
The electronic toll collection system is an important part of the intelligent transportation system. The microstrip antenna is the bridge between the OBU and RSU when they exchange information. This paper introduces the microstrip antenna's theory and simulation. The microstrip antenna's structure is simple and its operation band is in the $5.8 \mathrm{GHz}$. Its VSWR is less than 1.5 and the gain can reach $3.4 \mathrm{~dB}$. It makes the information exchanging between the OBU and RSU more accurate and morerapid
\end{abstract}

Keywords-ETC ; Intelligent transportation; Microstrip antenna ; $O B U$

\section{INTRODUCTION}

The electronic toll collection system is a new type of toll collection system with short name ETC. It is used in the highway, bridge and tunnel toll systems. Especially in the United States, Europe and Japan, the ETC has been utilized for many years and their technology has become very mature. The ETC has the following function: first, the car is not asked to stop when it passes the toll station; second, you should not to pay cash and the system will help you to complete the work; at last, when the ETC is used, it will help the toll station to save manpower through all the work be completed by the machine. Our country enacted the new standard about the ETC in 2007; this standard not only fills the gap in the standard of ETC system, but also will give us a reference when we use it. The ETC system consists of two parts, one is the Road Side Unit, short name RSU, which is placed in the top of high-speed toll station; another is the On Board Unit, short name OBU, which is placed on the windshield of the car. When the car passes the high-speed toll station, the RSU and OBU will exchange the information and complete transaction automatically, thus the time will be saved ${ }^{[2]}$. The microstrip antenna is an important component of OBU and RSU, which is the bridge of information exchange between OBU and RSU, and it guarantees the information can be received completely and quickly.

As a type of antenna, the microstrip antenna has been vigorously promoted and applied in the field of wireless communication $^{[5]}$. It has the features of small size, light weight and easy to integration. All of these characteristics comply with the RF antenna's design requirements. In the practical application, if the antenna radiation power is too strong, it will not only affect the adjacent lane's normal transaction, but also affect itself in the signal receiving and cause disturbance. In this paper, to meet our requirements we have designed a new type of microstrip antenna that has the features of low sidelobe and circular polarization.

\section{THE DESIGN OF ANTENNA}

According to different classification criteria, the antenna can be divided into different types. According to the structural characteristics, the microstrip antenna can be divided into two categories; microstrip patch antenna and microstrip slot antenna. According to the shape classification criteria, the microstrip antenna includes the following types: rectangular, circular, and annular and so on ${ }^{[1]}$. Also, the antenna's material, price and craftsmanship should be considered. Our goals are high performance, low price and simple production. In this paper the side-fed antenna with a cutting angle is selected as the microstrip antenna. It includes the following section: patch antenna, dielectric substrate and 1/4 wave impedance converter. Therefore, we should consider those factors in order to design a new microstrip antenna that has the functions of low sidelobe and circular polarization, and ensure the information can be received completely and quickly and people can enjoy its efficient service.

The design of the antenna is divided into the following steps:

1. Design the antenna main body and obtain the approximate value of the size of the antenna.

2. Realize the circular polarization and get the approximate value of the cutting angles.

3. Adjust the impedance of microstrip patch to match the dielectric substrate and calculate all the parameters.

4. Simulate the behavior of the antenna and get accurate values of the microstrip antenna.

\subsection{The Antenna Body Design}

Microstrip antenna can be divided into two kinds: when the patch shape is a long strip, it is a microstrip dipole antenna; when the patch is a unit of area, it is a microstrip antenna ${ }^{[3]}$. In this paper the second type is utilized. The microstrip antenna's plane structure is shown in Figure 1. The main body of the microstrip antenna is a rectangular patch structure with two cutting angles; with working frequency in $5.83 \mathrm{GHz}$.

As a part of the antenna, dielectric substrate has a signification effect on the information exchange ${ }^{[9]}$. A correct material means a good start. Considering those factors, we 
should select a proper material in order to design a high performance antenna. There are many kinds of dielectric substrate material, such as polytetrafluoroethylene, FR-4, ROGERS and so on ${ }^{[4]}$. When dielectric substrate material is selected not only the near field's characteristic is considered, but also the performance of the far field is paid close attention to. At the same time the price of the material and its craftsmanship should also be considered. According to the above statements and software simulation, the ROGERS is selected. Its length is set as L0, width W0, thickness $h$ and area s.

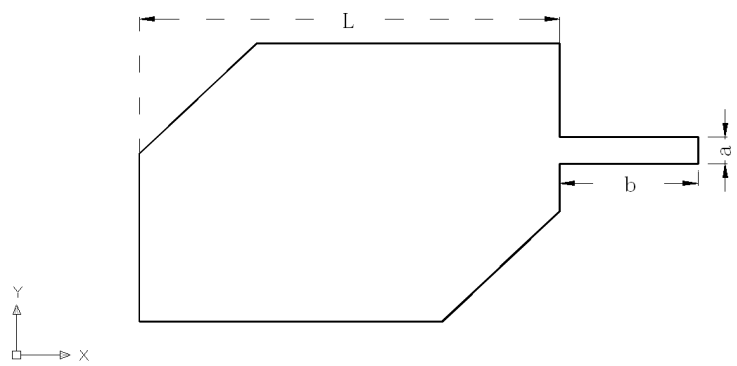

Figure 1 The microstrip antenna's plane structure

The formula to calculate the parameters of microstrip antenna patch and dielectric substrate are as the following.

$$
\begin{gathered}
L=\frac{c}{f \sqrt{\varepsilon_{\mathrm{e}}}} \\
\mathcal{E}_{e}=\frac{\varepsilon r+1}{2}+\frac{\varepsilon_{r}-1}{2}\left(1+12 \frac{h}{a}\right)^{-\frac{1}{2}} \\
L 0=L+0.2 \lambda_{g} \\
W 0=L+0.2 \lambda_{g} \\
\lambda_{g}=\lambda_{0} / \sqrt{\varepsilon_{r}}
\end{gathered}
$$

In the above formula, $\varepsilon_{e}$ is the effective dielectric constant and $\lambda_{0}$ is spatial wavelength. According to the above formula the approximation values of L, L0 and W0 can be calculated, and these parameters will be used in the software simulation. All the parameters will be optimized and the optimal dimensions of the dielectric substrate will be obtained in the process of simulation. The results will be used to set the final size of the antenna.

\subsection{Realization of Circular Polarization}

There are many kinds of circular polarization antenna, such as single feedback circular polarization microstrip antenna, microstrip antenna array, orthogonal feedback circular polarization microstrip antenna, and so on. Microstrip antenna has two kinds of circular polarization modes. One is the lefthand circular polarization and another is the right-hand circular polarization. There are many ways to realize antenna's circular polarization. An angle or a chink can be cut in order to produce two orthogonal resonances - the TM01 and TM02, and they have the same amplitude with phase difference $\pi / 2^{[8]}$. Based on the cavity model theory, the single side feedback rectangular patch antenna can be used to produce two degenerate modes with the same amplitude, but it can not generate 90 degree phase difference. A separating element in the microstrip antenna should be added to separate the two resonant frequencies in order to generate circular polarized wave with 90 degree phase difference. At last the antenna's circular polarization is realized by this way.

The advantage is that the extra circuit is not asked to produce circular polarization and the antenna's structure is simplified. All of them are easy to implement. In this paper the method of cutting angles in the opposite corner in the patch is used to realize the circular polarization. First, we should cut 2 identical isosceles right triangles in the opposite corner, their length is $\mathrm{c}$ and area is $\Delta \mathrm{s}$. The relationship between $\mathrm{s}$ and $\Delta \mathrm{s}$ is

$$
\left|\frac{\Delta s}{s}\right|=\frac{1}{2 Q}
$$

And the $\mathrm{Q}$ is the quality factor. Then, the relationship between $\mathrm{C}$ and $\mathrm{L}$ can be obtained through the formula (1) and (6). The relationship is

$$
c=0.35 L
$$

At last, the antenna that has been cut angles is made to match the $50 \Omega$ through $1 / 4$ wavelength impedance transformer.

\subsection{Impedance Matching Design}

The 1/4 wavelength impedance transformer is utilized to match the impedance of microstrip patchb in order to make the return loss become smaller, to optimize VSWR and reduce the loss of antenna. It can be divided into the following types: a single impedance converter, double impedance converter, triple impedance converter and multisection impedance converter. In this paper the single frequency impedance has meet our requirement.

In the paper the width of microstrip is set a, the length is set b. Because of the $1 / 4$ wavelength impedance transformer is a narrow band microstrip line, approaching zero, its width can be negligible, so the value of a is near a rate of 0 . The value of $\varepsilon e$ can be obtained through this formula.

$$
\varepsilon_{e}=\frac{\varepsilon_{r}+1}{2}+\frac{\mathcal{E}_{r}-1}{2} \bullet\left\{\left[1+12\left(\frac{W}{H}\right)^{-1}\right]^{-1}+0.04\left(1-\frac{W}{H}\right)^{2}\right\}
$$

According to the above formula the value of the parameter can be obtained, at the same time the approximation value of the parameters can be obtained through calculation, and these parameters can be used in the software simulation. In the process of simulation, all the parameters should be optimized in order to get the optimal dimensions of the $1 / 4$ wavelength impedance transformer. The results will be as the final size of the antenna. 


\section{THE Simulation RESULT}

In this paper the Ansoft HFSS V13.0 is used be as the simulation software. All the value of antenna's parameter can be obtained according to the above formula. When the value of the $\mathrm{L}$ is between $11.0 \mathrm{~mm}$ and $11.6 \mathrm{~mm}$, the performance of the antenna is achieved the best. If the size of the antenna is too small or too large, the working frequency of antenna will offset $5.83 \mathrm{GHz}$. In the paper, the value of the $\mathrm{L}$ is set $11.3 \mathrm{~mm}$.

All the value of the dimensions can be obtained through adjust and optimize the parameter. They are: $\mathrm{L}=11.3 \mathrm{~mm}$, $\mathrm{a}=0.9 \mathrm{~mm}, \quad \mathrm{~b}=7 \mathrm{~mm}, \quad \mathrm{c}=4 \mathrm{~mm}, \mathrm{~h}=1.5 \mathrm{~mm}, \quad \mathrm{~L} 0=25 \mathrm{~mm}$, $\mathrm{W} 0=17 \mathrm{~mm}$. Antenna optimized diagram is shown in Figure 2 .

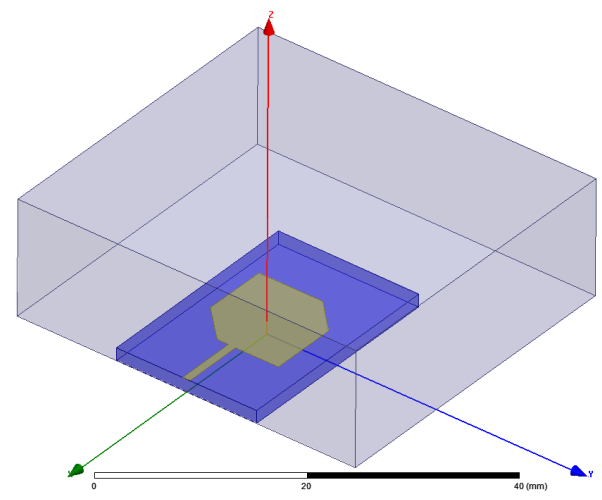

Figure 2 The diagram of antenna

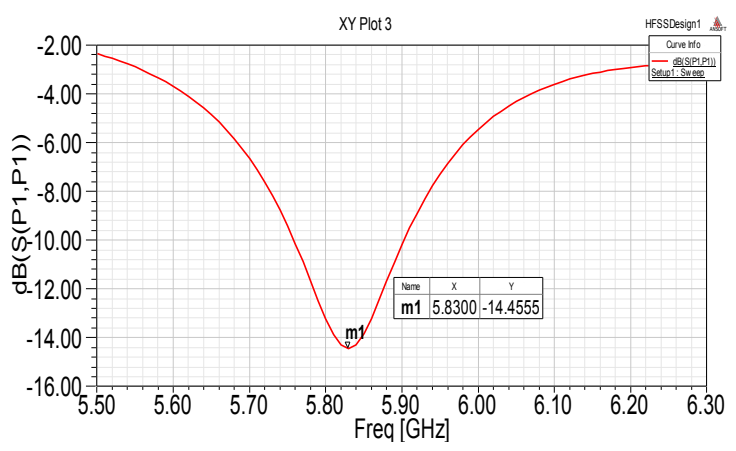

Figure 3 The return loss of antenna

Figure 3 is the S11 curves of the microstrip patch antenna. It indicates the function of the antenna's emission efficiency ${ }^{[10]}$. The value of S11 reflects the return loss of microstrip antenna and the compatibility of the antenna. If the value is too large, it means the reflect energy is greater and this antenna's emission efficiency is too worse.

As show in the Figure 3, the pad value of the antenna is $14.45 \mathrm{~dB}$ at the frequency of $5.83 \mathrm{GHz}$ and the value is meet the national standard's requirement, so it is reasonable in the design of S11.

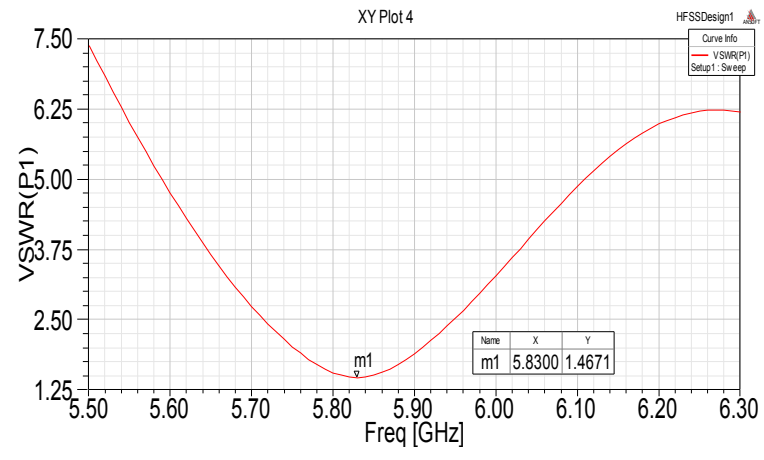

Figure 4 The antenna's VSWR curve

The antenna's VSWR simulation curve is as shown in Figure 4. VSWR is the short name of Voltage Standing Wave Ratio; it reflects the transmission loss of the antenna ${ }^{[6]}$. The value of VSWR is too large, the reflected power is too large and the transmission efficiency is too low. The maximum value of VSWR is 1.5 in the national standard. As show in the figure 4 , the value of VSWR is 1.46 at the frequency of $5.83 \mathrm{GHz}$; it is less than the requirements of the national standard, so the design is reasonable. Because of the value is close to the maximum value, it should be optimized continuously in order to make the antenna impedance matching between each part to achieve the best effect.

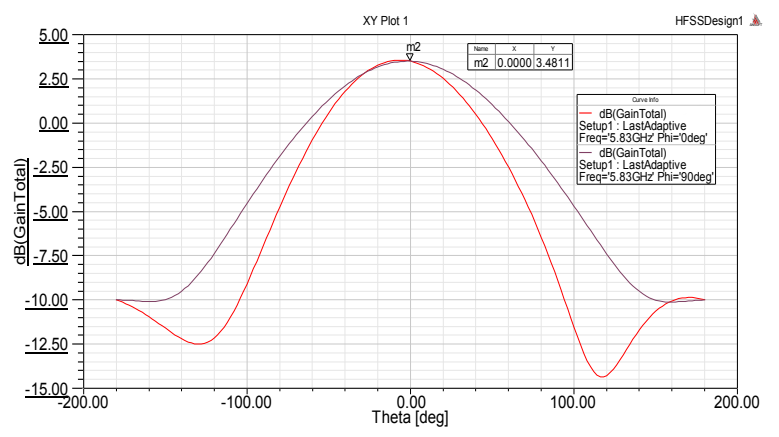

Figure 5 The gain of the antenna

The antenna gain is the ratio of output power from an amplifier system to the input power. It reflects the concentrated radiation level of input power. The gain of antenna is showed in Figure 5. The antenna gain is $3.48 \mathrm{~dB}$ at the frequency of $5.83 \mathrm{GHz}$.

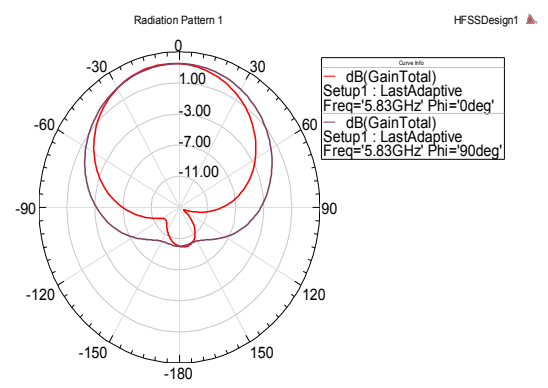

Figure 6 The direction of E plan

There is a close relationship between the gain and the antenna mainlobe. The thinner the mainlobe, the higher the 
gain $^{[7]}$. The direction of E plan is as show in Figure 6, the backlobe is small enough in the horizontal antenna surface. It makes OBU can corresponding with RSU in normal circumstances and don't interfere with adjacent lane and disturb the other OBU. At the same time it accelerates the vehicle passing rate and improves the work efficiency of high-speed toll station.

From the above simulation results, the microstrip antenna working frequency is $5.83 \mathrm{GHz}$, its gain is $3.4 \mathrm{~dB}$, return loss is $-14.45 \mathrm{~dB}$ and the VSWR is less than 1.5 , all of these parameters are meet the requirement of national standard. The back-lobe is small enough and there is no sidelobe in the antenna sidelobe chart. Above all, these antenna parameters meet the design requirements and can be used in production.

\section{CONCLUSION}

This paper introduces a type of microstrip antenna's design that is used in the ETC system. Its working frequency is $5.83 \mathrm{GHz}$, the value of $\mathrm{S} 11$ can get $-14.15 \mathrm{~dB}$ and the VSWR is 1.46, all of them accord with the requirement of national standards. At the same time the gain of antenna can get $3.4 \mathrm{~dB}$ when it works at $5.83 \mathrm{GHz}$, and its back-lobe is small enough and there is no sidelobe in the E plane. These are also accord with the requirement of the ETC system. Also, it can be continued to optimism and adjust the antenna's parameters in order to get a high performance antenna.

\section{REFERENCES}

[1] John Klaus, The antenna. Beijing: Electronic Industry Press.2011 .

[2] GB/T20851.1 - 2007. Beijing: China Standard Press.2007.

[3] Yongjun Xie, Ying Liu. Principle and engineering application HFSS. Beijng: China Social Sciences Publishing House.2009.

[4] Mingyang Li, Min Liu, Fang Yang. The design of HFSS. Beijing: Electronic Industry Press.2011

[5] Shunshi Zhong. the theory and technology of antenna. Beijing: Electronic Industry Press.2011.

[6] Shoukang Fan,Jin Li,Rong Hu. Microwave technology, microwave circuits and antennas. Beijing: Mechanical Industry Press.2009

[7] Jijie Yin. Microwave technique and antenna. Beijing: Electronic Industry Press.2009

[8] Yipeng Shao,Ming Bai,Huijin Ma. " 5.835GHz microstrip array antenna used in ETC ". Modern Electronics Technique.2012.pp. 148-151.

[9] Jing Li,Dingguo Xu,Shengxiang Cheng. "Research on the RFID-based Small Circularly Polarized Antenna". Electronic Technology.2009.pp. 16-19.

[10] Xiaoliang Shao,Lijuan Zhao, Yongqing Zou. "Design of A Novel Singlefeed Broadband Circularly Polarized Microstrip Antenna". Journal of microwaves.2008.pp.21-24. 